\title{
Experience STORIES: A Visual News Search and Summarization System
}

\author{
Ilija Subašić and Bettina Berendt \\ Department of Computer Science, K.U. Leuven, Belgium
}

\begin{abstract}
Using data collections available on the Internet has for many people become the main medium for staying informed about the world. Many of these collections are dynamic by nature, evolving as the subjects they describe change. We present the STORIES system for (a) learning an abstracted story representation from a collection of time-indexed documents; (b) visualizing it in a way that encourages users to interact and explore in order to discover temporal "story stages" depending on their interests; and (c) supporting the search for documents and facts that pertain to the user-constructed story stages.
\end{abstract}

\section{Introduction}

Search engines, RSS feeds, micro-blogging tools, and many other services support Internet users in story tracking: following the developments of topics over time. This is usually done by manually or automatically issuing a series of same queries about an event ("Haiti Earthquake"), a person ("Britney Spears"), or a scientific area ("text mining"). This creates a challenge for information seekers because large numbers of new documents from different sources keep arriving at a fast rate. There is clearly a need for different search interfaces and search experience, which provide a concise abstracted representation of information from all the pertinent parts of a source or source set. Users will be able to profit most from summarising services that provide convenient interfaces to both the abstracted summary and the underlying documents, and that allow for and encourage a flexible, (inter)active exploration of the space of the abstracted "stories" and at the same time searches of the space of documents.

To enhance the story tracking search experience, we pursue two goals: (a) give users a more active role in the search process, and (b) break away from traditional "top-10" ranked document search interfaces. As a part of this we present the STORIES system for news-stories tracking, which instantiates these ideas. It consists of story learning (done by the system) and graphical support for story understanding and story search (provided to the user). The paper builds on the detailed explanation in [1] it describes a re-implementation with new interface features and examples from a new corpus.

\section{Related Work}

Apart from "classical" news search engines like Google News or Yahoo! News, recently many alternative ways of tracking and browsing news collections have been

J.L. Balcázar et al. (Eds.): ECML PKDD 2010, Part III, LNAI 6323, pp. 619 623, 2010.
(C) Springer-Verlag Berlin Heidelberg 2010 
developed. Google News Timeline (http://newstimeline.googlelabs.com/) provides a pre-set time period (day, week, month, year) overview of news using a timeline interface. It allows for the tracking of news sources, arbitrary queries or entities such as movies, books, music... Another Google system, named Fast Flip (http://fastflip.googlelabs.com/), provides an interface for browsing news articles resembling hard-copy newspaper reading. The Yahoo! Correlator (http://correlator.sandbox.yahoo.net/) associates a search term with all its related "events". EMM NewsExplorer(http://emm.newsexplorer.eu) and EMM NewsBrief (http://emm.newsbrief.eu) are news search and summarization services tracking news from a number of multi-lingual sources. The SearchPoint (http://searchpoint.ijs.si/) system allows users to focus on a specific sub-topic of search results. MemeTracker (http://www.memetracker. . org/) tracks quotes from news and visualises their "burstiness" using interactive charts. These deployed systems rest on a growing body of work on topic detection and tracking, temporal text mining, and visual web search surveyed in [1].

In contrasts to these other tools, our system combines visual search, summarization, and burst-pattern detection into a single interface which provides an interactive inspection of temporal changes in a corpus.

\section{Method}

First, a corpus of text-only documents is transformed into a sequence-of-terms representation. Subsequently, basic term statistics are calculated to identify candidates for story basics. We applied different measures to obtain the story basics including the top ranked words based on term frequency, TF.IDF weight, combining "regular" terms with terms referencing some named entities, and all terms form a corpus.

For story understanding, we analyse a text corpus and its (user-definable) time-indexed subsets. For each time-indexed subset of the whole corpus $c_{i}$, the frequeny of the co-occurrence of all pairs of content-bearing terms $b_{j}$ in documents is calculated as the number of occurrences of both terms in a window of $w$ terms, divided by the number of all documents in $c_{i}$. This measure of frequency and therefore relevance is normalised by its counterpart in the whole corpus $C$ to yield time relevance as the measure of burstiness: $T R_{i}\left(b_{1}, b_{2}\right)=$ $\left(\operatorname{freq}_{i}\left(b_{1}, b_{2}\right)\right) /\left(\right.$ freq $\left._{C}\left(b_{1}, b_{2}\right)\right)$. Thresholds are applied to avoid singular associations in small sub-corpora and to concentrate on those associations that are most characteristic of the period and most distinctive relative to others: This gives rise to the story graphs $G_{i}=\left\langle V_{i}, E_{i}\right\rangle$ for time periods $i$. The edges $E_{i}$ of $G_{i}$ are the story elements: all pairs $\left(b_{1}, b_{2}\right)$ with absolute frequencies and $T R$ above the respective thresholds. The nodes $V_{i}$ of $G_{i}$ are the story basics, the terms involved in at least one association in this symmetric graph: $\left\{b_{j} \mid \exists b_{k}:\left(b_{j}, b_{k}\right) \in E_{i}\right\}$. From each document, we extract sentences containing "facts", short statements with semantic role labelling, as returned by Open Calais (http://www.opencalais.com/). The full set of these sentences for each 


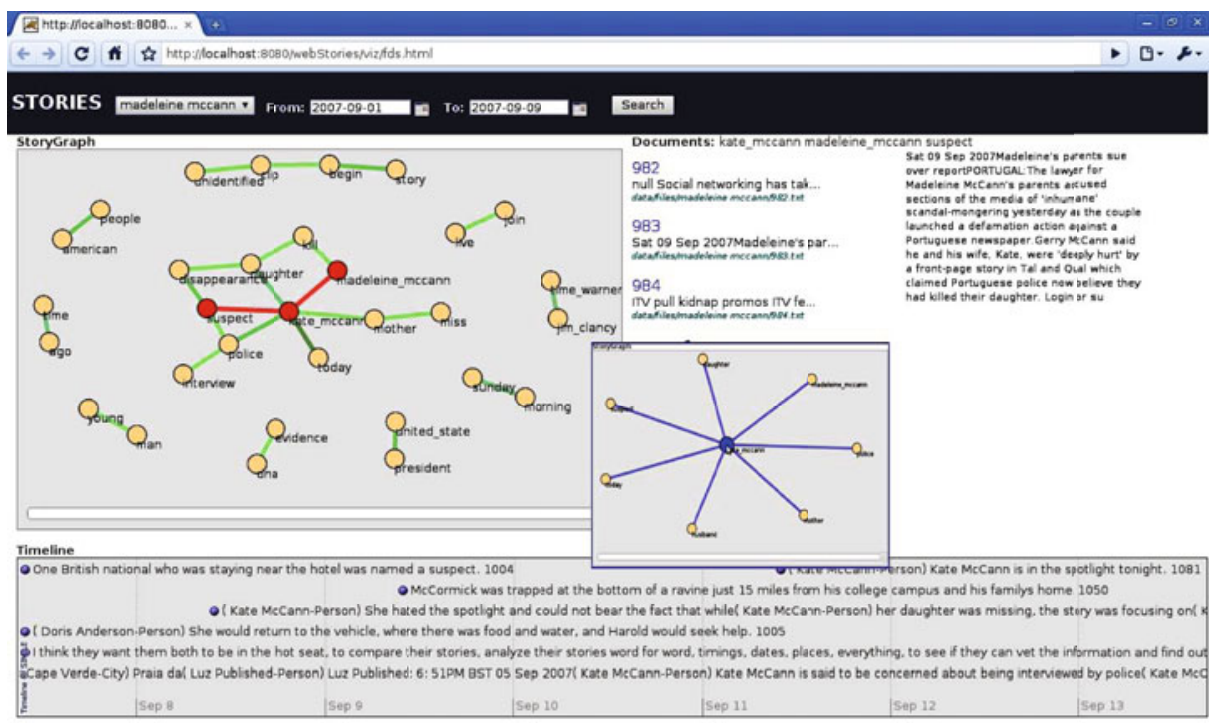

Fig. 1. Web interface: A story graph (left) is built based on articles about the disappearance of a person. By marking the edges connecting the person's name (top node of the subgraph marked in red) with another name (middle node) as a "suspect" (left node), the user obtains a list of pertinent documents (centre), whose text can be inspected (right). The timeline (bottom) shows the important "facts" from a selected time period. The overlaid tracking story graph shows how the search can be focused on the chosen node over different time periods.

time period is indexed using Lucene (http://lucene.apache.org). We then use story graphs to filter the most important facts: for each of the graph's edges, we query the index, using node names of the edge as query terms, and select the top sentences as defined by Lucene. We treat the resulting set of short textual statements as a summary of the story.

Story search can be constrained by the nodes of a subgraph of the story graph. Retrieval is then restricted to documents relevant to these subgraphs. The selection of documents of the starting corpus $C$ corresponds to a top-level query; this query is expanded by the information from the subgraph and the time restriction. STORIES then uses all the nodes $n$ as a query (restriction) for the documents inside $c_{i}$ to obtain the pertinent document subset, as identified by a search over a Lucene index.

\section{Tool}

Figure 1 shows a screenshot of our STORIES front-end. We apply the method to news articles obtained from different sources on the Web. Corpora can be compiled either on a continuing basis (e.g., subscribed-to feeds) or in response to a top-level query to a search engine. Our indexing service crawls a number 
of news aggregators and retrieves documents for a given top-level query. The top-level query describes the whole story (e.g., "Haiti Earthquake" or "person name" for crime cases or celebrity reporting). Data cleaning and other data preparation steps are then applied, in particular HTML wrapper induction and removal, tokenisation, cross-document named-entity recognition, lemmatisation, and stopword removal. Finally, document and term measures as described in the previous section are computed. The system backend is developed in Java and the front-end using GUESS (http://graphexploration.cond.org/) library for the stand-alone version, and a JavaScript implementation of the spring-layout algorithm for the web version of the tool.

The primary representations are visualisations of story graphs. They provide functionalities to: (1) scan over time to track the global story evolution, (2) zoom in and out by time, by adapting the period-window size, (3) zoom in and out by detail, uncovering more details about the story by setting the $T R$ values (a configurable colour coding schema of edges points to the different values of time relevance), (3) tracking certain terms or entities in time, by selecting the corresponding node. This outputs a graph of bursty co-occurrences including this "tracking node" as its central node.

By clicking on a single edge, the user can select documents associated with the term pair. For easier and more flexible search, users may also select an edge and then highlight a subgraph which contains the selection's adjoining edges and neighbouring nodes. Each selected edge expands the query and restricts the document set based on it. In this way, the user incrementally builds the query and at the same time can discover and learn about the story. Search provides a list of documents, and a set of sentences visualized along the time-line using one day as an atomic period. This allows users to interact with the story using different views: patterns, sentences ("facts"), and documents.

\section{Evaluation}

Evaluations of search quality demonstrated that STORIES finds coherent subsets of documents, that its quality is comparable to or better than state-of-the-art clustering, and that the tool enables people to answer questions on groundtruth events accurately and quickly [1]. We also compared our method with other "bursty-pattern discovery" methods, with a framework that leverages sentence retrieval and internal pattern structure and evaluates the sentences against a ground truth. Our experiments showed that different methods perform at similar levels overall, but provide distinctive advantages in some settings [2. In a third, ongoing round of evaluations we perform a user study evaluating how users can discover information using our and other search interfaces.

\section{Outlook}

In future work, we will investigate more advanced language processing (linguistic parsing, semantic role labelling for story graphs, etc.), the use of lexical 
resources and other background knowledge, as well as different sources of media bias/viewpoints. We also plan to explore aggregation and analysis dimensions other than time, such as multilinguality. Further quantitative and qualitative evaluations will be carried out.

\section{References}

1. Subašić, I., Berendt, B.: Discovery of interactive graphs for understanding and searching time-indexed corpora. Knowledge and Information Systems 23(3), 293 319 (2010)

2. Subašić, I., Berendt, B.: From bursty patterns to bursty facts: The effectiveness of temporal text mining for news. To appear in Proc. ECAI'2010 (2010) 